\title{
Proteção das Pessoas nos Esquemas de ligaçÃo À Terra "TN" e “IT"
}

\section{Resumo}

O correto dimensionamento dos dispositivos de proteção das pessoas contra contactos indiretos em instalações elétricas de Baixa Tensão (BT), é uma das condições fundamentais para que uma instalação possa ser utilizada e explorada com conforto e em perfeitas condições de segurança.

De acordo com a normalização em vigor, é, também, uma das condições essenciais para a certificação ou licenciamento das instalações elétricas por parte das entidades ou organismos responsáveis, a quem estão atribuídas estas competências.

A função dos dispositivos de proteção das pessoas contra os contactos indiretos será o corte automático da alimentação da instalação elétrica, que em caso de defeito, e em consequência do valor e da duração da tensão de contacto, evitará o risco de se produzirem efeitos fisiopatológicos perigosos nas pessoas.

As medidas de proteção adotadas obrigam à coordenação entre os Esquemas de Ligação à Terra (ELT)) adotados, as características dos condutores de proteção e os respetivos dispositivos de proteção.

Neste artigo são apresentados alguns exemplos de cálculo dos dispositivos de proteção das pessoas contra contactos indiretos, de acordo com o ELT adotado para a instalação elétrica.

\section{Cálculos no Esquema de Ligação à Terra "TN"}

O ELT TN caracteriza-se por todas as massas da instalação serem ligadas ao ponto da alimentação ligado à terra, próximo do transformador ou do gerador da alimentação da instalação, por meio de condutores de proteção.
O ponto da alimentação ligado à terra é, em regra, o ponto neutro.

Nas instalações fixas pode-se utilizar um só condutor com as funções de condutor de proteção e de condutor neutro (designado por condutor PEN) desde que o condutor de proteção tenha uma secção não inferior a $10 \mathrm{~mm}^{2}$, se de cobre ou a $16 \mathrm{~mm}^{2}$, se de alumínio e, a parte da instalação comum (esquema TN-C) não esteja localizada a jusante de um dispositivo diferencial.

Este ELT encontra-se representado na Figura 1.

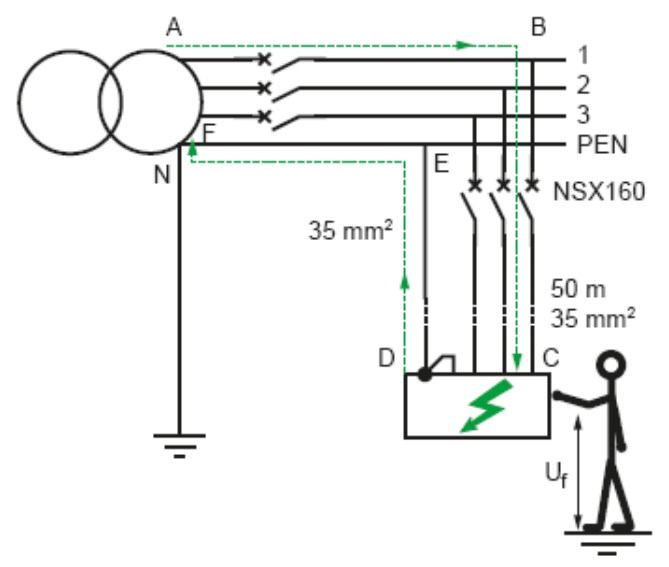

Figura 1. Esquema de ligação à terra " TN" (Fonte Schneider Electric)

Neste ELT um defeito de isolamento é similar a um curtocircuito fase neutro, e o corte deve ser assegurado pelo dispositivo de proteção contra curtos-circuitos, com um tempo máximo de corte especificado que é função da tensão limite convencional $\left(U_{L}\right)$ admissível para o local da instalação, ou seja, $25 \mathrm{~V}$ ou $50 \mathrm{~V}$ em corrente alternada, sendo o valor definido pela classificação do local quanto às influências externas.

Segundo a norma CEI 364 o tempo de corte do dispositivo de proteção deverá ser de $0,4 \mathrm{~s}$ para $\mathrm{U}_{\mathrm{L}}=50 \mathrm{~V}$ e $0,2 \mathrm{~s}$ para $\mathrm{U}_{\mathrm{L}}=25 \mathrm{~V}$. 
Seguidamente, apresenta-se um circuito de uma instalação elétrica de BT, trifásica (400V), onde é adotado o ELT TN-C, ou seja, a função de neutro e de proteção estão combinadas num único condutor (PEN).

Este circuito é apresentado na figura 2.

O circuito tem um comprimento de $40 \mathrm{~m}$, a secção do condutor de fase é de $95 \mathrm{~mm}^{2}$ e a do condutor de proteção é de $50 \mathrm{~mm}^{2}$. O circuito está protegido com disjuntor NS $250 \mathrm{~N}$ (Schneider Electric) equipado com disparador magnetotérmico TM 250 curva D.

Pretende-se verificar se neste ELT, a proteção das pessoas contra contactos indiretos está efetivamente garantida com este dispositivo de proteção.

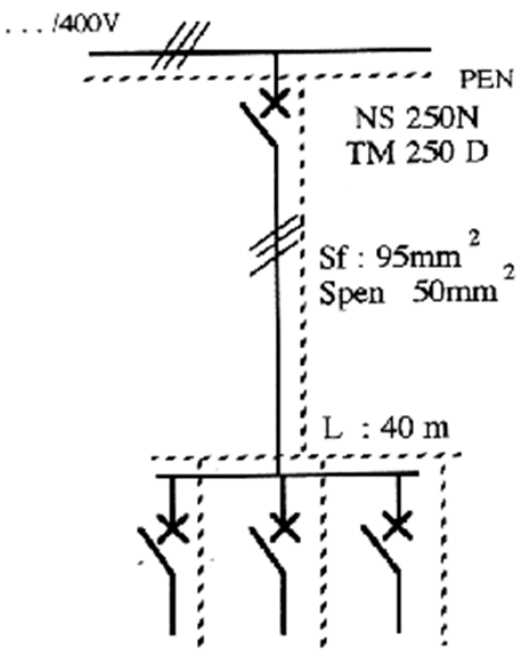

Figura 2. Exemplo de cálculo.

\section{Esquema de ligação à terra TN-C}

Uma condição fundamental para o correto dimensionamento do dispositivo de proteção, é conhecer a curva de atuação do dispositivo, de maneira a obter-se o valor da corrente correspondente ao limiar de funcionamento do disparador magnético do aparelho de proteção.

A curva deste dispositivo de proteção é apresentada na figura 3.

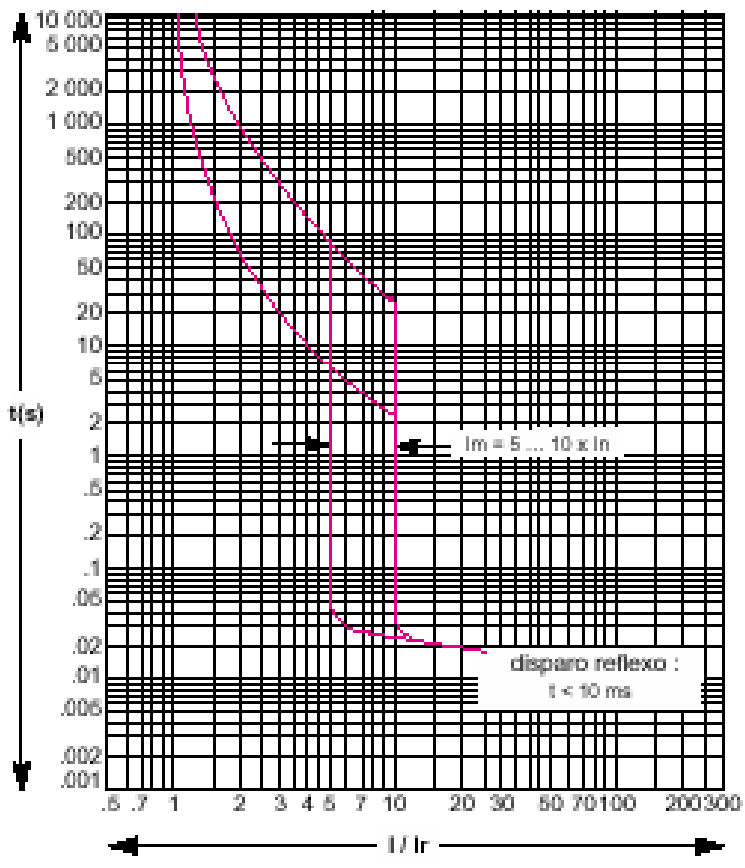

Figura 3. Curva de disparo TM250D

(Fonte: Schneider Electric)

Como se pode verificar, a atuação do disparador magnético deste disjuntor poderá ser regulada para funcionar entre 5 a 10 vezes o valor nominal (In), ou seja, entre 1250 e $2500 \mathrm{~A}$.

Neste ELT a impedância da malha de defeito Zs será:

$Z_{s}=\frac{K \times U_{0}}{I_{d}}$

em que $\mathrm{K}$ toma o valor de 0,8 para instalações eléctricas, $U_{0}$ é a tensão simples nominal da instalação e $I_{d}$ é a corrente de defeito.

Para que a protecção contra curtos-circuitos também garanta a protecção contra contactos indirectos, é necessário para os disjuntores que:

$Z_{s} \leq \frac{K \times U_{0}}{I_{m}}$

em que $I_{m}$ é a corrente de actuação do disparador magnético do dispositivo. 
Para a protecção por fusíveis, é necessário que:

$Z_{s} \leq \frac{K \times U_{0}}{I_{f}}$

em que $\mathrm{I}_{\mathrm{f}}$ é a corrente convencional de funcionamento do fusível.

Atendendo a que neste ELT um defeito é efectivamente um curto-circuito entre uma fase e o condutor de protecção, a impedância da malha de defeito será então:

$$
Z_{s} \approx R_{s}=\rho_{f} \times \frac{l}{s_{f}}+\rho_{P E} \times \frac{l}{s_{P E}}
$$

em que $\rho_{\mathrm{f}}$ é a resistividade de condutor de fase, $\rho_{\mathrm{PE}}$ a resistividade do condutor de protecção, I é o comprimentos dos condutores, $s_{f}$ a secção do condutor de fase e $S_{P E} a$ secção do condutor de protecção.

Considerando que os condutores de fase e de protecção têm as mesmas características, a impedância da malha de defeito será então:

$Z_{s} \approx R_{s}=\rho \times \frac{l}{s_{f}} \times(1+m)$

em que;

$$
m=\frac{s_{f}}{s_{P E}} \quad \text { e } \quad \rho=1,25 \times \rho_{20^{\circ} \mathrm{c}}
$$

O comprimento máximo protegido do circuito será então, para disjuntores:

$l \leq \frac{K \times U_{0} \times s_{f}}{\rho \times(1+m) \times I_{m}}$

e para fusíveis será de:

$l \leq \frac{K \times U_{0} \times s_{f}}{\rho \times(1+m) \times I_{f}}$
Para o circuito apresentado na figura 2, o comprimento máximo protegido do circuito, para uma regulação do disparador magnético de $5 x \ln (\operatorname{Im}=1250 A)$ será de:

$l \leq \frac{0,8 \times 230 \times 95}{0,0225 \times(1+19) \times 1250} \leq 214 m$

para uma regulação do disparador magnético de 10x/n (Im=2500A) será de:

$l \leq \frac{0,8 \times 230 \times 95}{0,0225 \times(1+19) \times 2500} \leq 107 m$

Atendendo que o comprimento do circuito é de $40 \mathrm{~m}$, verifica-se que em qualquer dos casos o disjuntor garante a protecção das pessoas contra contactos indirectos.

No entanto, deve-se também verificar se o tempo de actuação do dispositivo é compatível com o especificado pelas curvas de segurança, para a tensão limite convencional definida para o local da instalação, que como já foi referido, segundo a norma CEI 364 deverá ser de $0,4 \mathrm{~s}$ para $\mathrm{U}_{\mathrm{L}}=50 \mathrm{~V}$ e, $0,2 \mathrm{~s}$ para $\mathrm{U}_{\mathrm{L}}=25 \mathrm{~V}$.

Assim, torna-se importante calcular o valor da tensão de contacto em caso de defeito.

$U_{c}=R_{P E} \times I_{d}$

em que:

$I_{d}=\frac{K \times U_{0}}{Z_{s}}=\frac{K \times U_{0}}{\rho \times \frac{i}{s_{f}} \times(1+m)}$

então:

$U_{c}=R_{P E} \times \frac{K \times U_{0} \times s_{f}}{\rho \times l \times(1+m)}$

$R_{P E}=\rho \times \frac{l}{s_{P E}}$

$U_{c}=K \times U_{0} \times \frac{m}{1+m}$ 
Para o exemplo em consideração, representado na figura 2, tem-se:

$U_{c}=0,8 \times 230 \times \frac{1,9}{1+1,9}=120,6 \mathrm{~V}$

Pelas curvas de segurança, e para a tensão limite convencional de $25 \mathrm{~V}$, o dispositivo deve actuar num tempo inferior a $180 \mathrm{~ms}$.

Como se pode verificar na curva de funcionamento do disjuntor, apresentada na figura 3, o dispositivo actuará num tempo inferior ao referido e compatível com o especificado pela norma CEI 364.

Assim, para esta instalação, e para este ELT, pode-se garantir que o disjuntor apresentado protege efectivamente as pessoas contra contactos indirectos.

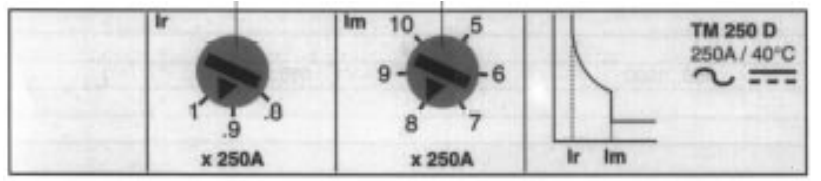

Figura 4. Painel de regulação do relé eletrónico TM250D

(Fonte Schneider Electric)

\section{Cálculos no esquema de ligação à terra "IT"}

Este ELT apresenta como principal vantagem, a garantia de continuidade de serviço em presença de um primeiro defeito de isolamento.

Como se explicou no artigo publicado na revista anterior, neste esquema de ligação à terra, a presença de um primeiro defeito não origina valores de tensão de contacto perigosos para as pessoas.

No entanto, é obrigatório a presença de um Controlador Permanente de Isolamento ( $\mathrm{CPI}$ ), de maneira a sinalizar o defeito e permitir a sua eliminação o mais rapidamente possível.

A manifestação de um segundo defeito, sem que tenha sido eliminado o primeiro, implicaria agora a existência de tensões de contacto muito perigosas, devendo ser tomadas as medidas adequadas de forma a evitar riscos de efeitos fisiopatológicos perigosos nas pessoas suscetíveis de ficar em contacto com partes condutoras simultaneamente acessíveis. Como tal, a proteção das pessoas neste ELT é orientada para o dimensionamento dos dispositivos de proteção atuarem na situação de segundo defeito.

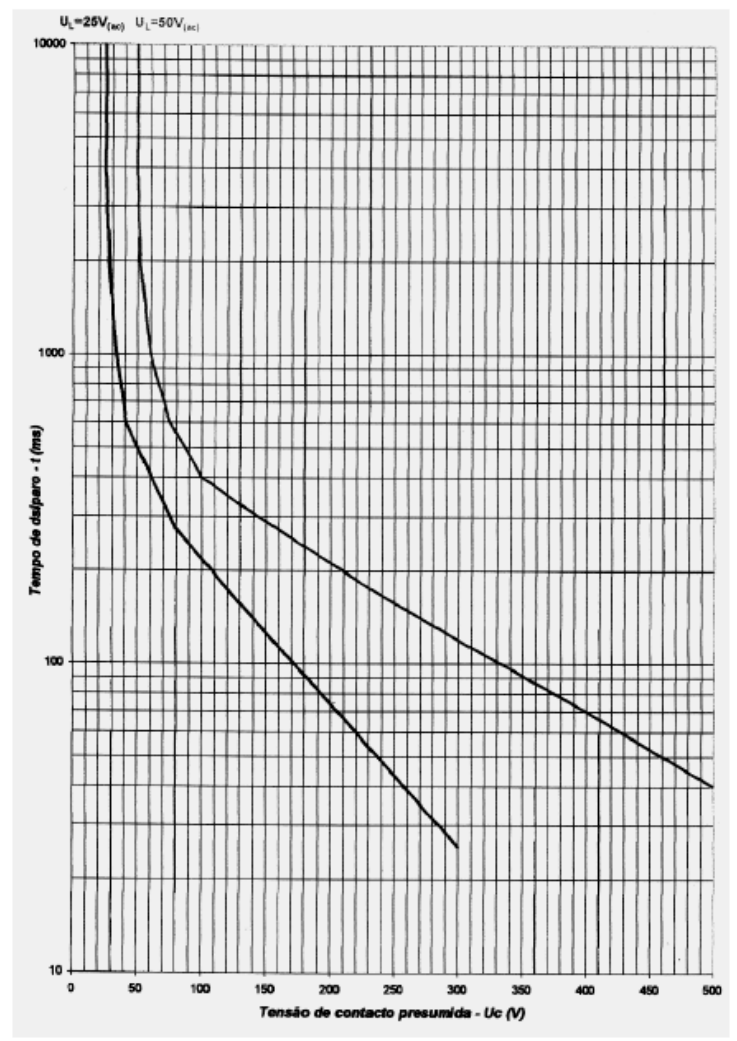

Figura 5. Curvas de segurança em instalações de baixa tensão

É importante eliminar todas as situações que possam contribuir para diminuir a fiabilidade do sistema. Assim, não se deve distribuir o condutor neutro, pois poderá correr-se o risco de manifestar-se um segundo defeito sem que o primeiro tenha sido sinalizado, atuando a proteção e perdendo-se todas as vantagens inerentes à utilização deste ELT.

Este esquema caracteriza-se por as partes ativas da instalação elétrica serem isoladas da terra ou ligadas a esta através de uma impedância de valor elevado. As massas dos aparelhos de utilização são ligadas à terra, individualmente ou por grupos. 
A situação mais comum nas instalações onde é adotado este ELT, é todas as massas, incluindo as da fonte, estarem ligadas a um mesmo elétrodo de terra.

A figura 6 apresenta a malha de defeito na situação de um primeiro defeito na instalação. Assim, as condições de eliminação da corrente de um segundo defeito é então garantida pelas mesmas condições indicadas para o esquema TN.

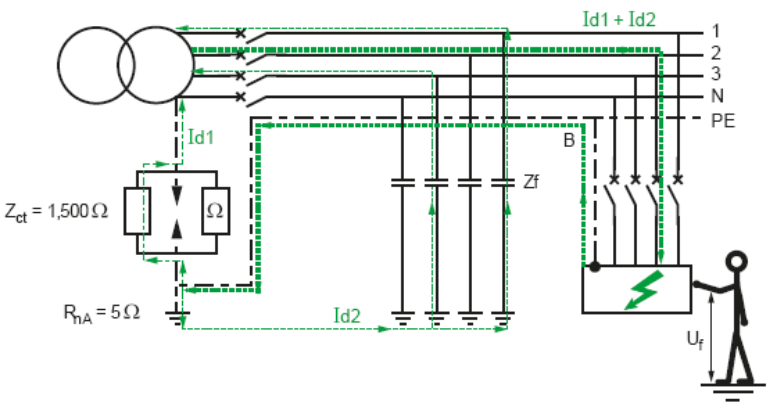

Figura 6. Esquema de Ligação à Terra IT:10 defeito (Fonte Schneider Electric)

A figura 7 apresenta a malha de defeito na situação de um segundo defeito sem o primeiro ter sido eliminado.

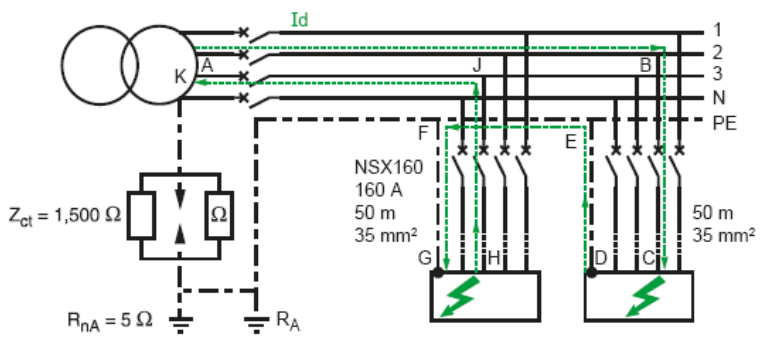

Figura 7. Esquema de Ligação à Terra IT:20 defeito

(Fonte Schneider Electric)

Neste esquema IT, a proteção das pessoas contra contactos indiretos é fundamentalmente garantida por dois tipos de equipamentos:

- pelos $\mathrm{CPI}$, essencialmente destinados à vigilância do primeiro defeito, embora possam também ser utilizados como dispositivos de proteção nas situações em que for necessário provocar o corte ao primeiro defeito;
- pelos dispositivos de proteção contra sobreintensidades (disjuntores e fusíveis). Estes dispositivos são utilizados nas situações em que ao segundo defeito são aplicadas as condições de proteção definidas para o esquema TN;

Seguidamente, apresenta-se um circuito de uma instalação elétrica de BT, trifásica (400V), onde é adotado o esquema IT, sem neutro distribuído (situação comum neste ELT) . Este circuito é apresentado na figura 8.

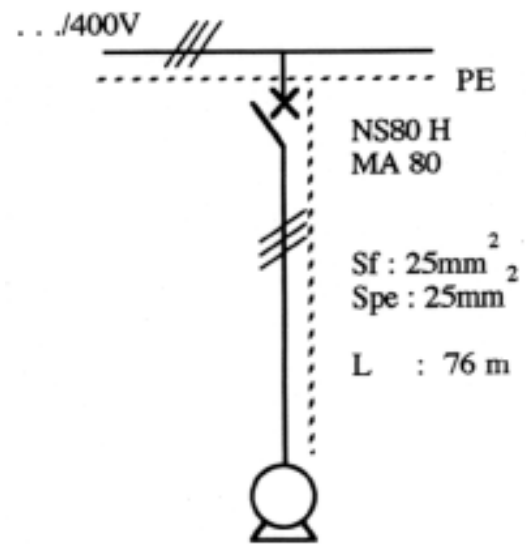

Figura 8: Exemplo de cálculo. Esquema IT

O circuito tem um comprimento de $76 \mathrm{~m}$, a secção do condutor de fase e de proteção é de $25 \mathrm{~mm} 2$.

O circuito está protegido com disjuntor especifico para proteção de saídas motor NS $80 \mathrm{H}$ (Schneider Electric) equipado com disparador "motor" integrado MA 80. Pretende-se verificar se neste esquema de ligação à terra, a proteção das pessoas contra contactos indiretos está efetivamente garantida com este dispositivo de proteção.

Também no caso deste esquema é fundamental para o correto dimensionamento do dispositivo de proteção, conhecer a curva de atuação do dispositivo, de maneira a obter-se o valor da corrente correspondente ao limiar de funcionamento do disparador magnético do aparelho de proteção.

A curva deste dispositivo de proteção é apresentada na figura 9. 
Como se pode verificar, a atuação do disparador magnético deste disjuntor verifica-se entre 6 a 14 vezes o valor nominal (In=80A), ou seja, entre 480 e 1120A.

Também neste esquema de ligação à terra, tal como no regime TN, um defeito é efetivamente um curto-circuito entre uma fase e o condutor de proteção.

Então, para este circuito, sem neutro distribuído, a impedância da malha de defeito será:

$$
Z_{s} \leq \frac{K \times \sqrt{3} \times U_{0}}{I_{m}}
$$

em que $I_{m}$ é a corrente de actuação do disparador magnético do dispositivo.

Neste ELT considera-se como boa aproximação que ao segundo defeito, o comprimento da malha de defeito é duplo em relação ao primeiro defeito.

Então, a impedância da malha de defeito será neste caso:

$$
Z_{s} \approx R_{s}=2 \times\left(\rho_{f} \times \frac{l}{s_{f}}+\rho_{P E} \times \frac{l}{s_{P E}}\right)
$$

Considerando também que os condutores de fase e de protecção têm as mesmas características, a impedância da malha de defeito será então:

$$
Z_{s} \approx R_{s}=2 \times\left(\rho \frac{l}{s_{f}} \times(1+m)\right)
$$

em que ;

$m=\frac{s_{f}}{s_{P E}}=1 \quad$ e $\quad \rho=1,25 \rho_{20^{\circ} c}$

O comprimento máximo protegido deste circuito será então, para disjuntores:

$l \leq \frac{K \times \sqrt{3} \times U_{0} \times s_{f}}{2 \times \rho \times(1+m) \times I_{m}}$

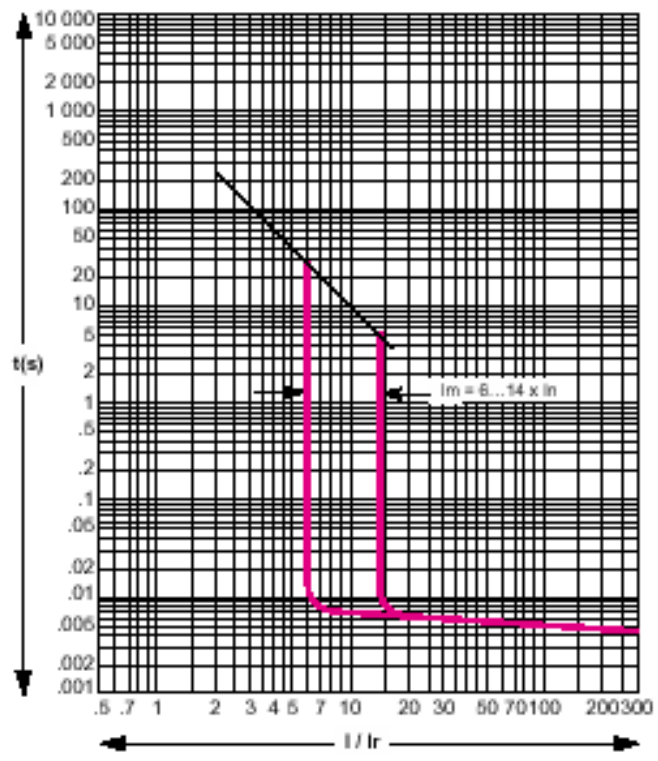

Figura 9: Curva de disparo MA80

(Fonte Schneider Electric)

Para o circuito apresentado na figura 8, o comprimento máximo protegido do circuito, para uma regulação do disparador magnético de $6 x \ln (I \mathrm{Im}=480 A)$ será de:

$l \leq \frac{0,8 \times \sqrt{3} \times 230 \times 25}{2 \times 0,0225 \times(1+1) \times 480} \leq 184 m$

Para uma regulação do disparador magnético de 14xIn (Im=1120A) será de:

$$
l \leq \frac{0,8 \times \sqrt{3} \times 230 \times 25}{2 \times 0,0225 \times(1+1) \times 1120} \leq 79 m
$$

Atendendo que o comprimento do circuito é de $76 \mathrm{~m}$, verifica-se que para qualquer regulação do disparador MA (6 a $14 x(n)$, o disjuntor garante a protecção das pessoas contra contactos indirectos.

No entanto, tal como no esquema de ligação à terra TN, também se deve verificar se o tempo de actuação do dispositivo é compatível com o especificado pelas curvas de segurança, para a tensão limite convencional definida para o local da instalação, que como já foi referido, segundo a norma CEI 364 deverá ser de $0,4 \mathrm{~s}$ para $\mathrm{U}_{\mathrm{L}}=50 \mathrm{~V}$ e, $0,2 \mathrm{~s}$ para $\mathrm{U}_{\mathrm{L}}=25 \mathrm{~V}$. 
Assim, torna-se importante calcular o valor da tensão de contacto em caso de segundo defeito.

$U_{c}=R_{P E} \times I_{d}$

em que, através de uma dedução idêntica à efetuada para o esquema de ligação à terra TN, obtêm-se:

$U_{c}=K \times \sqrt{3} \times U_{0} \times \frac{m}{2 \times(1+m)}$

Para o exemplo em consideração, representado na figura 6, tem-se:

$U_{c}=0,8 \times \sqrt{3} \times 230 \times \frac{1}{2 \times(1+1)}=79,7 \mathrm{~V}$

Pelas curvas de segurança (figura 10), e para a tensão limite convencional de $25 \mathrm{~V}$, o dispositivo deve atuar num tempo inferior a $280 \mathrm{~ms}$.

Como se pode verificar na curva de funcionamento do disjuntor, apresentada na figura 9, o dispositivo atuará num tempo inferior ao referido e compatível com o especificado pela norma CEI 364.

Assim, também para esta instalação, e para este ELT, podese garantir que o disjuntor apresentado protege efetivamente as pessoas contra contactos indiretos.

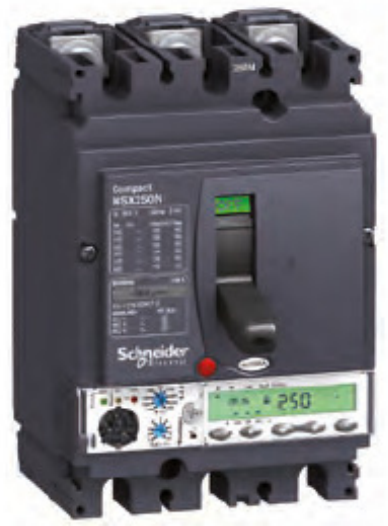

Figura 10. Disjuntor Compact NS $80 \mathrm{H}$ com disparador MA (Fonte Schneider Electric)

\section{Conclusões}

Neste artigo apresentou-se dois exemplos de cálculo e dimensionamento dos dispositivos de proteção das pessoas contra contactos indiretos. Um exemplo para o ELT "TN", e outro para o ELT "IT".

Atendendo a que nestes esquemas, e para o caso dos exemplos apresentados, uma situação de defeito é sempre uma situação de curto-circuito entre um condutor ativo e a massa do equipamento de utilização, ou seja, um curtocircuito entre um condutor ativo e o condutor de proteção, são, normalmente, os dispositivos de proteção contra sobreintensidades que terão a função de também garantir a proteção das pessoas contra contactos indiretos.

Na realidade, nos exemplos que são apresentados, o que se teve que fazer foi verificar se realmente o dispositivo de proteção contra curtos-circuitos também verificava as condições necessárias à proteção das pessoas contra contactos indiretos.

Este facto foi analisado através da verificação do máximo comprimento protegido.

Efetivamente, nestes dois esquemas de ligação à terra, para se poder dimensionar corretamente os dispositivos de proteção, é fundamental conhecer bem as características do circuito, nomeadamente comprimento da instalação, tipo de condutores, trajeto dos cabos, secção dos condutores.

Outro fator importante, é verificar se o dispositivo atua num tempo compatível com especificado pelas normas de segurança. Este facto depende das condições do local da instalação elétrica. De acordo com estas condições, os regulamentos impõe como tensão de contacto limite, $25 \mathrm{~V}$ ou $50 \mathrm{~V}$.

Assim, torna-se importante calcular o valor da tensão de contacto em caso de defeito e, através da curva de segurança dos $25 \mathrm{~V}$ ou $50 \mathrm{~V}$, conforme o caso, obter o tempo 
máximo de atuação do dispositivo de forma a que a tensão de contacto nunca ultrapasse o valor da tensão limite convencional. Este facto obriga, também, a conhecer muito bem as curvas de funcionamento dos dispositivos de proteção, para verificar se esta regra do tempo de atuação também é garantida.

No caso dos disjuntores, a zona de funcionamento magnético dos disparadores é quase instantânea, não sendo a regra do tempo de atuação problemática para este tipo de equipamento de proteção.

O facto torna-se mais importante quando os dispositivos de proteção são fusíveis.

\section{Bibliografia}

- "Esquemas de Ligação à Terra e a Proteção de Pessoas". Aulas do Professor José António Beleza Carvalho , Instituto Superior de Engenharia do Porto.

- Regras Técnicas das Instalações Elétricas de Baixa Tensão

- Instalações Elétricas Industriais" - João Mamede Filho Editora LTC 5a Edição

- "Esquemas de Ligação à Terra em BT (Esquemas de ligação à terra)" Caderno Técnico no 172 - Bernard Lacroix e Roland Calvas. Edição: Schneider Electric.

- Electrical-Installation-Guide_2016. Edição: Schneider Electric

Divulgação:

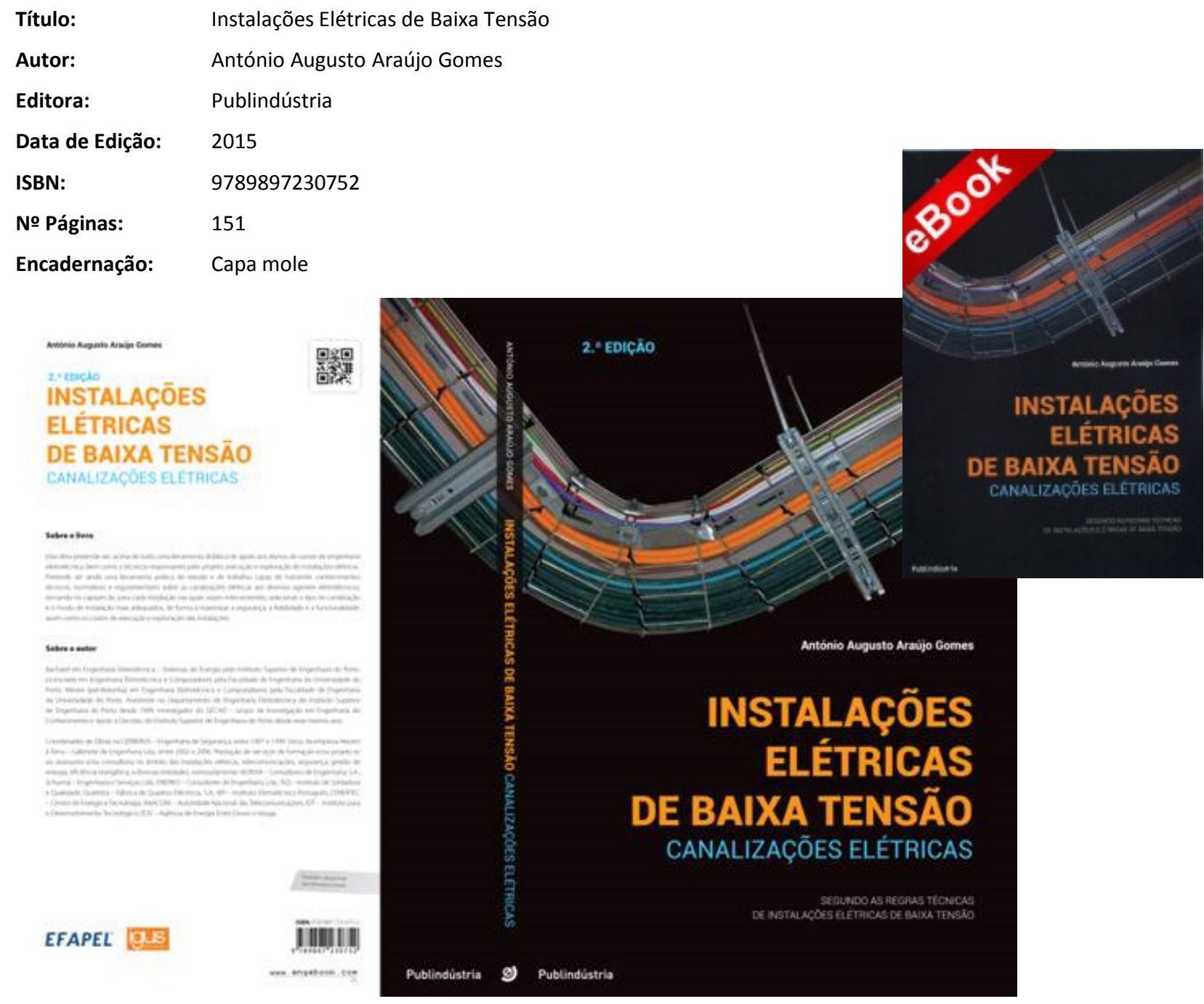

